the possible risks of aortic leaflet injury caused by the knots. Subsequently, the diseased section of the lateral wall along the incision was excluded, and the incision was closed in a double-layered fashion with felt strips to achieve PLV (Video E1). The aortic crossclamp time was 135 minutes, and the cardiopulmonary bypass time was 241 minutes. The postoperative course was uneventful, and the postoperative echocardiogram showed improved LV shape/ dimension and function (LVDd $=57 \mathrm{~mm}, \mathrm{LVEF}=30 \%$ ) with no mitral, tricuspid, or aortic regurgitation. She was discharged, able to walk, on postoperative day 49. Three years after the operation, she is doing well; her cardiac status is NYHA class I, LVDd is $56 \mathrm{~mm}$, and LVEF is $33 \%$.

\section{DISCUSSION}

To the best of our knowledge, this is the first report describing a case wherein dual SVRs were successfully performed for the reconstruction of the diseased basal septum and LV lateral wall in a patient with sarcoidosis-induced dilated cardiomyopathy.

Although cardiac sarcoidosis and idiopathic cardiomyopathy have many features in common, the important difference is that the former often involves multiple and patchy LV segments, most frequently in the anterior LV wall and interventricular septum. ${ }^{1}$ SVR is reported to be an effective therapeutic option for cardiac sarcoidosis. ${ }^{2}$ Further, in patients with both anterior and septal LV involvement, septal anterior ventricular exclusion is the method of choice in SVR because it offers low operative mortality and a favorable long-term outcome. ${ }^{2,3}$ Nevertheless, the septal anterior ventricular exclusion method is limited in that it inevitably requires an incision along the anterior $\mathrm{LV}$ wall for recon- struction of the interventricular septum ${ }^{3}$; thus, it can barely be applied in patients with a diseased septum and an intact anterior wall. In our case, the basal septum and the lateral wall were diseased and the anterior wall was intact.

A permanent AV sequential pacemaker may be necessary postoperatively when conduction disturbance is induced by the periannular stitches taken along the right coronary cusp; however, this is not an issue when the patient already has a pacemaker implanted or is willing to undergo implantation. In our case, complete AV block was already treated by permanent pacemaker implantation. In such cases, the surgeon can reconstruct the basal septum through the ventriculotomy at the lateral LV wall to achieve PLV without the concern of damaging the conduction system around the aortic annulus and consequently decrease the enlarged LV diameter while preventing possible future ventricular septal perforation.

In conclusion, we have reported a case wherein dual SVRs were successfully performed in a patient with cardiac sarcoidosis. This novel method wherein a patch is placed on the scarred basal septum through a ventriculotomy for PLV at the diseased lateral wall was effective in the surgical treatment of dilated cardiomyopathy involving multiple and patchy LV segments. The long-term outcome of this treatment remains unknown ${ }^{2,3}$; hence, a careful follow-up is mandatory.

\section{References}

1. Bargout R, Kelly RF. Sarcoid heart disease: clinical course and treatment. Int J Cardiol. 2004;97:173-82

2. Daimon M, Sasaki T, Woo E, Ozawa H, Morimoto T, Katsumata T. Successful surgical treatment for dilated cardiomyopathy with cardiac sarcoidosis. Ann Thorac Surg. 2007;84:e12-3.

3. Suma H, Isomura T, Horii T, Nomura F. Septal anterior ventricular exclusion procedure for idiopathic dilated cardiomyopathy. Ann Thorac Surg. 2006;82:1344-8.

\title{
Potential role of the Impella Recover left ventricular assist device in the management of postinfarct ventricular septal defect
} \author{
Michele La Torre, MD, ${ }^{\mathrm{a}}$ Turin, Italy \\ From the Cardiac Surgery Department, San Giovanni Battista Hospital, ${ }^{a}$ and the Car- \\ diac Surgery Department, Mauriziano Hospital, ${ }^{\mathrm{b}}$ Turin, Italy. \\ Impella Recover is a trade name of ABIOMED, Inc, Danvers, Mass. \\ Received for publication Jan 18, 2008; accepted for publication Feb 23, 2008. \\ Address for reprints: Francesco Patanè, MD, Divisione di Cardiochirurgia, Ospedale \\ San Giovanni Battista, C.so Bramante 88, Torino, Italia (E-mail: f_patane@ \\ hotmail.com). \\ J Thorac Cardiovasc Surg 2009;137:1288-89 \\ $0022-5223 / \$ 36.00$ \\ Copyright (c) 2009 by The American Association for Thoracic Surgery \\ doi:10.1016/j.jtcvs.2008.02.061
}

Francesco Patanè, MD, ${ }^{\mathrm{a}}$ Paolo Centofanti, MD, ${ }^{\mathrm{a}}$ Edoardo Zingarelli, MD, ${ }^{\mathrm{b}}$ Fabrizio Sansone, MD, ${ }^{\mathrm{a}}$ and
Postinfarction ventricular septal defect (VSD) remains a dangerous complication after acute myocardial infarction with regard to both natural history and surgical treatment. VSD appears in $1 \%$ to $2 \%$ of patients after a myocardial infarction. Prognosis is poor, with only $75 \%$ of untreated patients surviving after 24 hours, 50\% after 1 week, 30\% after 2 weeks, and only $15 \%$ after 30 days. ${ }^{1}$ The literature describes different outcomes depending on whether the corrective operation is performed promptly or deferred; mortality is $67 \%$ 
if surgery is performed in the first week after postinfarction VSD develops, $75 \%$ if in the second week, and $17 \%$ after that. $^{2}$

\section{CLINICAL SUMMARY}

The Impella Recover (ABIOMED, Inc, Danvers, Mass) is a small intracardiac axial-flow left ventricular assist device (LVAD) that provides 4 to $5 \mathrm{~L} / \mathrm{min}$ continuous blood flow. The device can be set at 10 different speeds, from 10,000 to $33,000 \mathrm{rpm}$. The Impella is advanced into the left ventricular cavity in a retrograde fashion through a prosthetic graft sutured to the ascending aorta (in the operating theater) or through the femoral artery (in the catheterization laboratory), thereby passing the aortic valve. ${ }^{3}$ The correct position, essential for the correct function of the device, can be verified by means of the differential pressure trace on the console, transesophageal echocardiography, or fluoroscopy.

Our objective has been to exploit characteristics of this device to maintain a stable hemodynamic condition in patients with postinfarct VSD, thus allowing us to postpone surgical closure of the defect for 3 to 4 weeks (changing the Impella Recover LVAD if necessary). During this period, myocardium around the defect becomes fibrotic and thus provide a better footing for a steady suture.

After the Impella implantation, a left ventricular overload reduction appears. In addition, left-to-right ventricular shunt decreases and may disappear.

To improve prognosis, is important to implant the Impella Recover LVAD before excessive worsening of the patient's hemodynamic condition. If the LVAD is not available, an intra-aortic balloon pump is implanted.

In our experience, after implantation of the Impella Recover with the $\mathrm{P} 4$ program level $(2.5 \mathrm{~L} / \mathrm{min})$ the pulmonary/systemic perfusion ratio is lowered, with an average reduction of 1 without reversal of the shunt to avoid systemic hypoxia risk. Ascending aortic pressures generally increase, with average increases of 10 to $15 \mathrm{~mm} \mathrm{Hg}$ in both systolic and diastolic pressures. Pulmonary arterial pressures in our experience decrease, with an average reduction of 35 to $40 \mathrm{~mm}$ $\mathrm{Hg}$. Wedge pressures also decrease, with an average reduction of 10 to $15 \mathrm{~mm} \mathrm{Hg}$. Moreover, in cases of mitral regurgitation there is a relevant reduction of incompetence from moderate to mild. Furthermore, in every case it has been possible to significantly reduce inotropic drug infusion, thus decreasing potential side effects. Other parameters controlled are the right ventricular stroke work index and the venous oxygen saturation.

After Impella Recover LVAD implantation, daily transthoracic echocardiography is performed for the first
5 days; after that, echocardiography is performed every 2 days as long as the patient is in stable condition. In the case of new hemodynamic worsening, we generally increase the program level $(\mathrm{P})$ on the console. Nevertheless, once the P7 or P8 program level has been reached, it is important to consider the possibility of an early operation. On the other hand, if the patient's hemodynamic condition remains stable, the operation can be deferred for 3 to 4 weeks, at which time there is less surgical difficulty in suturing the patch to thicker and more solid tissue to close the defect.

Surgical closure of the postinfarction VSD may not be possible, for example after a very wide infarct with a serious ejection fraction reduction. The Impella Recover LVAD can then be used as a bridge to transplant, as long as there are no contraindications. ${ }^{4,5}$

The management of postischemic VSD requires a simple protocol to stabilize hemodynamic condition in a short time. Mechanical support should be as practical and effective, as is the Impella Recover LVAD. This system is much less invasive than a complete peripheral bypass with oxygenation, such as extracorporeal membrane oxygenation, in terms of both the postoperative management and the duration of mechanical support.

This new surgical management protocol permits modification of the timing of the operation, potentially improving prognosis after postinfarction VSD. So our experience has been positive, if limited to a few cases. We report promising improvements in hemodynamic parameters. Pulmonary arterial pressure, right ventricular stroke work index, venous oxygen saturation, wedge pressure, transesophageal echocardiographic evaluation of the shunt, and mitral incompetence (if present) are all useful indicators of the patient's condition. Our objectives in the future will be to characterize hemodynamic and echocardiographic cutoffs to evaluate efficacy of the device and thus decide in a given case whether to continue with LVAD support, close the defect immediately, or evaluate the patient for cardiac transplant.

\section{References}

1. Kouchoukos NT, Karp RB, Blackstone EH, Doty DB, Hanley FL. Cardiac surgery. 3rd ed. Philadelphia: Churchill Livingstone; 2003. p. 456-71.

2. Di Summa M, Actis Dato G, Centofanti P, Fortunato G, Patanè F, Di Rosa E, et al. Ventricular septal rupture after a myocardial infarction: clinical features and long term survival. J Cardiovasc Surg (Torino). 1997;38:589-93.

3. Siegenthaler MP, Brehm K, Strecker T, Hanke T, Nötzold A, Olschewski M, et al The Impella Recover microaxial left ventricular assist device reduces mortality for postcardiotomy failure: a three-center experience. J Thorac Cardiovasc Surg. 2004; 127:812-22.

4. Meyns B, Vanermen H, Vanhaecke J, Sergeant P, Daenen W, Flameng W. Hemopump fails as bridge to transplantation in postinfarction ventricular septal defect. J Heart Lung Transplant. 1994;13:1133-7.

5. Patanè F, Zingarelli E, Sansone F, Rinaldi M. Acute ventricular septal defect treated with an Impella recovery as a 'bridge therapy' to heart transplantation. Interact Cardiovasc Thorac Surg. 2007;6:818-9. 